\title{
Quieter and Greener rotorcraft: concurrent aerodynamic and acoustic optimization
}

\author{
Gunther Wilke ${ }^{1}$ D
}

Received: 25 November 2020 / Revised: 17 February 2021 / Accepted: 31 March 2021 / Published online: 21 April 2021

(c) The Author(s) 2021

\begin{abstract}
Within the DLR project VicToria an aerodynamic and aero-acoustic optimization of helicopter rotor blades is performed. During the optimization, three independent flight conditions are considered: hover, cruise and descent flight. The first two flight conditions drive the power requirements of the helicopter rotor, while the descent flight is the loudest flight condition for current helicopter generations. To drive down the required power and the emitted noise, a multi-objective design approach coupled with surrogate models is utilized to find a Pareto optimal set of rotors. This approach allows to identify the trade-offs to be made when laying emphasis on either goal function. The underlying CFD simulations utilize fourth-order accurate spatial schemes to capture the vortex dominated flow of helicopter rotor blades. The paper presents the validation of the setups, the optimization results and the off-design analysis of a chosen set of blades from the Pareto front. The conclusion is that the utilization of the Pareto front approach is necessary to find good rotor designs, while the utilization of high-order methods allows for efficient CFD setups.
\end{abstract}

Keywords Rotorcraft $\cdot$ Multi-disciplinary optimization $\cdot$ Surrogate-based optimization $\cdot$ Multi-objective

\section{Introduction}

Helicopters play a unique role among all aircraft due to their universal and versatile mission applicability. Due to their ability to hover, they can perform tasks that other aircraft cannot. However, this comes at a price. The fuel consumption of helicopters for traveling specific distances is larger than for regular aircraft, the ability to hover is energy consuming and on top of that their noise generation is high. Thus, DLR is committed to decrease the overall fuel consumption of the helicopter throughout the mission envelope as well as the noise emission of the helicopter. One difficulty in the optimization of helicopter rotor blades lies in the rather different flight conditions the helicopter undergoes during a mission.

From previous research within the CleanSky 1 project [1], it has been seen that trade-offs have to be made between a low-power rotor in hover and a low-power rotor in forward

Gunther Wilke

gunther.wilke@dlr.de

1 Institute of Aerodynamics and Flow Technology Helicopter Department, Lilienthalplatz 7, 38108 Braunschweig, Germany flight. In DLR's VicToria project [2], the scope has been extended to also minimize the noise emission in descent flight, which is one of the noisiest flight conditions [3] for helicopters.

A good overview for the optimization of helicopter rotor blades is given by Ganguli [4]. Currently, there are two major routes taken by most researchers: the first one utilizes gradient-based optimization with either low-fidelity tools to quickly evaluate finite differences or high-fidelity tools using the adjoint formulation to efficiently obtain the necessary gradients. Recent examples that utilize high-fidelity CFD are given by Fabiano and Mavriplis [5], and Wang et al. [6]. Both of them adjoint the whole multi-disciplinary process: from the rotor comprehensive code, the CFD solver up to the acoustic post-processing tool. Fabiano and Mavriplis utilize 2.3 million grid points and 95 design variables, while Wang et al. optimized 92 design variables on a mesh with seven million grid points. These examples are among the first to also have an adjoint formulation for the unsteady flight conditions of the helicopter. Often simplifications are employed, where elasticity and unsteadiness are neglected, such as Garcia et al. [7] do for their tiltrotor optimization. Yet, they employ 6.2 million grid points for their rotor setup. The advantage of this approach is the little number 
of function evaluations in combination with the relatively little cost to evaluate the gradients. They all finished their respective optimization with 30 or less cycles.

Opposing the first route, the surrogate-based approach is often utilized, when either the formulation of the adjoint is not given or true multi-objective and multi-disciplinary optimizations are to be carried out. Roca León et al. [8] show a hybrid approach, where a gradient-based optimization is used to find the Pareto front of the problem. In the hover flight condition, they have the adjoint solution available, but in forward flight, they utilize surrogate models to efficiently obtain the gradients. Their approach is based on the Nash game, which allows finding a continuous Pareto front using a gradient optimizer. Johnson and Barakos built a computational chain for the rotor blade optimization using an artificial neural network and a genetic algorithm [9]. Surrogate models also lend themselves to combine different fidelities, such as Leusink et al. [10] or Wilke [11] do. The idea is to first seek out the design space with a low-fidelity tool and then only use a few high-fidelity samples to find the overall optimal location.

While most of this work is aerodynamic centered, Desvigne et al. [12] coupled aerodynamic and structural goal functions to perform a multi-disciplinary optimization. They, however, rely on the low-fidelity tools during their optimization and a noticeable offset in the hover performance is given when they re-evaluate their selected optimal blade with CFD.

Ganguli [4] also states the need to correctly model the physics of helicopter blades. Smith et al. [13] validate their CFD tools using the data generated by the HART II wind tunnel campaign. Their conclusion is that to correctly model the vortex dominated flow of helicopter rotor blades, either many grid points are necessary or higher order methods in space, or even both. Kowarsch et al. [3] match a flight experiment within $1 \mathrm{~dB}$ effective perceived noise level (ePNL) by simulating the complete helicopter with a sixth-order spatially accurate scheme and 192 million mesh points.

The current paper contributes to the ongoing research by optimizing three concurrent goal functions: the required power in hover and forward flight, as well as the noise emission in descent flight. Additionally, three constraints are included. Two of them try to consider the structural and dynamic behavior of the blade. In contrast to the otherwise so far used second-order solutions during optimization, fourth-order accurate CFD is carried out. The paper is structured as followed: first, the simulation methodology is described followed by a brief validation against various rotor blades. The multi-objective surrogate-based optimization setup is introduced and the optimization results are presented. An off-design analysis of a selected set of rotor blades is discussed, before the paper is closed with a summary and outlook for future work.

\section{Simulation methodology}

The overall simulation process is sketched in Fig. 1. Given a design vector from the optimizer (Powerful-OptimizationToolkit with Surrogate Models [11]), the preprocessing is started. This includes filling in necessary data for the comprehensive code HOST, but also the mesh generation with the in-house blade-grid generator $G^{3}$. The mesh generator utilizes transfinite interpolation [14] to quickly generate structured meshes of the same topology, here of O-O type. The comprehensive code then computes an initial trim state using simplified aero-dynamics as well as the blade deformation. The deformation is then passed onto the CFD solver FLOWer, which computes the high-fidelity aeroloads. These are then passed back to HOST, which then recomputes the helicopter trim and deformations. This is iterated until converged. The aero-acoustic post-processing tool APSIM then computes the emitted noise based on acoustic pressure surfaces obtained from FLOWer. From the different tools, the final goal function metrics are then abstracted and passed back to the optimizer for the next iteration.

\subsection{Comprehensive code HOST}

The comprehensive code HOST [15] has been originally developed by Eurocopter (now Airbus Helicopters). It employs the blade-element theory based on airfoil look-up tables to compute the aero-dynamic loads on the helicopter rotor. The rotor blade deformations are computed with a beam model assuming that the blade is long and slender. The multi-body dynamics are then coupled together using the

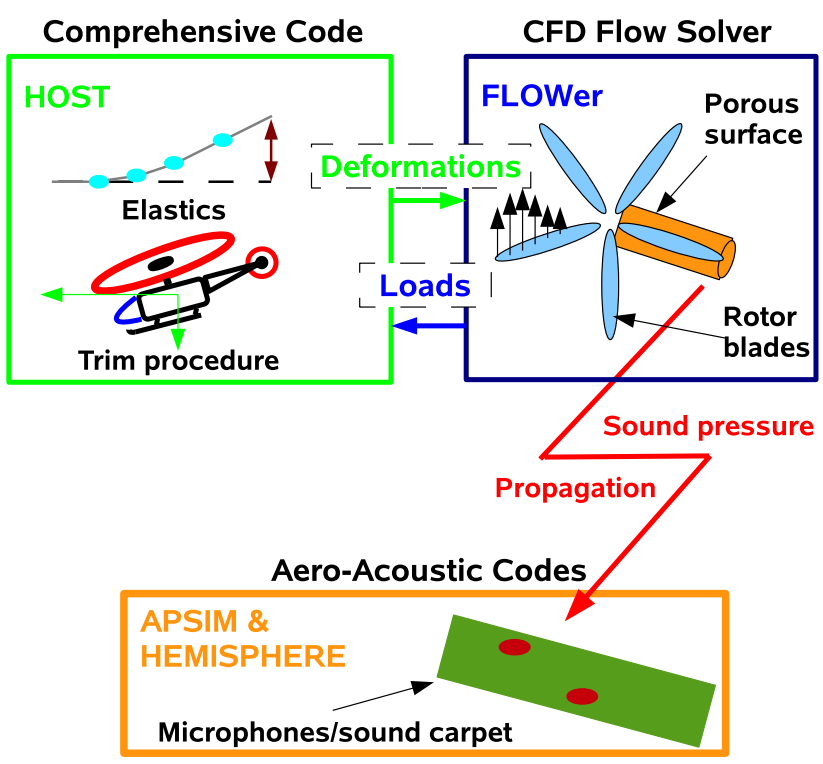

Fig. 1 Coupled simulation flow 
Lagrange equations. Coupling external airloads into HOST is done using the delta airloads approach [16].

\subsection{Flow solver FLOWer}

The legacy flow solver FLOWer [17] originally developed by DLR is utilized in this study. FLOWer is a multi-block structured flow solver. It uses an explicit five-stage Runge-Kutta scheme with implicit residual smoothing to advance the inner iterations of the dual-time stepping scheme of second order. The spatial scheme applied here is an upwind scheme [18] by Kitamura and Shima, which uses a fourth-order accurate reconstruction by Yamamoto et al. [19]. To further reduce numerical dissipation, the minmod limiters have been exchanged with van Albada type limiters. The SA-R turbulence model by Dacles-Mariani et al. [20] is used for closure of the U-RANS equations. Laminar-turbulent transition is empirically predicted on the rotor blades according to mechanisms chosen by Heister [21]. The Chimera approach [22] combines various grid blocks to allow them to move in arbitrary motion to each other.

\subsection{Acoustic-postprocessor APSIM}

The acoustic program APSIM [23] is used to predict the noise at locations far away from the rotor blades based on their surface pressures. Alternatively, if shock waves are on the surface, a porous surface may be applied instead [24]. The advantage is that the transport of the acoustic pressure is much more efficient using this Farrasat 1a [25] approach than to resolve these in CFD.

\section{Validation of simulation setups}

Before the optimization results are presented, the according simulation setups are first validated for each flight condition to assess the quality of the simulations.

\subsection{Hover (out-of-ground)}

The hover flight condition allows simplifying the setup when assuming an isolated rotor. This allows only modeling one rotor blade and employing periodic boundaries in the CFD mesh. The fuselage could also be modelled like in the following setups, yet the associated cost in hover to do so is prohibitively expensive since the development of the wake requires roughly four times more time steps than in descent or cruise flight, while the mesh has to be extended further downstream for correct results. Three grid densities are investigated. The finest grid has 161 grid points in each of the chordwise, normal and radial direction of the blade block, which is encompassed by an extension block with

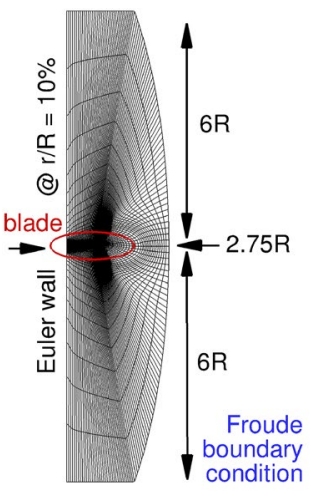

(a) hover

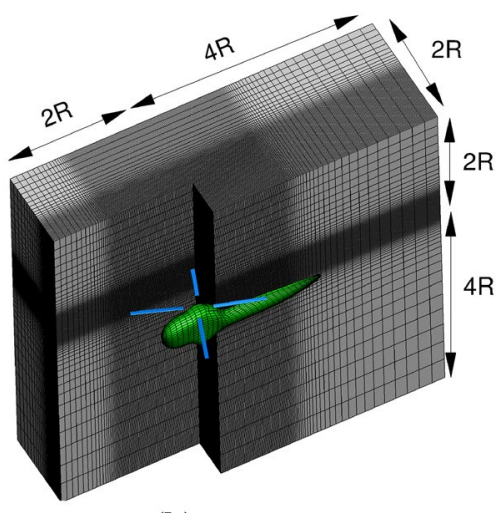

(b) cruise/descent
Fig. 2 Grid setups utilized for optimization (L2)

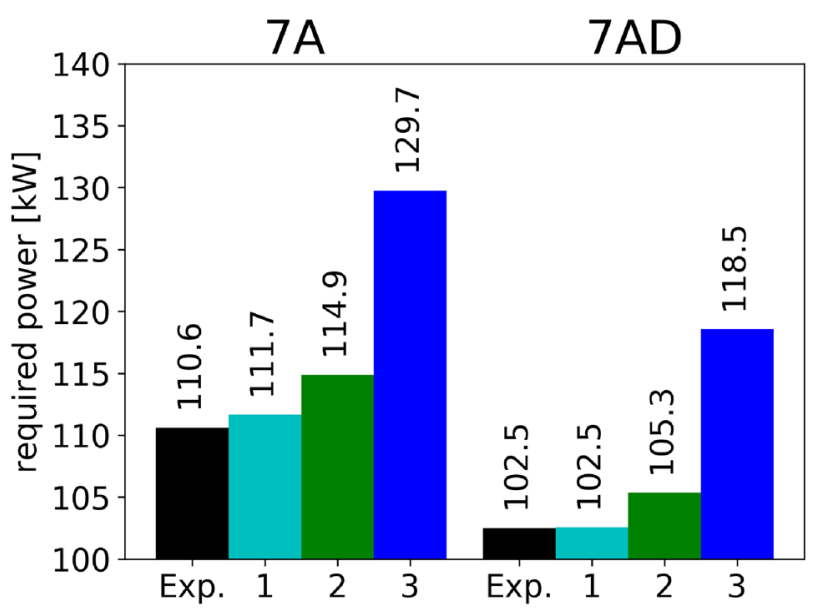

Fig. 3 Required power for 7A/7AD rotors in hover

$33 \times 33 \times 129$ grid points in the connecting side, normal, and radial direction. This leads to 4.4 million grid points of the finest mesh (L1). Leaving out every other grid point in each direction yields the level 2 grid (L2), and repeating this, the level 3 grid (L3) is obtained. The extension of the farfield is six rotor radii in the vertical and 2.75 radii in the horizontal direction, see Fig. 2a. A time step equivalent to $\Delta \psi=0.5^{\circ}$ of a rotation is chosen.

Two data points from the Helishape campaign [26] are selected. Data point 61 belongs to the 7A rotor, while data point 95 belongs to the 7AD rotor. In Fig. 3, the required power of the rotor is shown for the experiment along with the results for each grid level. While the L3 solution is far off from the experiment for both rotors, the L2 solution produces good results and the L1 solution matches closely with the experiment. Looking at the pressure distribution, Fig. 4, of the $7 \mathrm{~A}$ rotor at $\mathrm{r} / \mathrm{R}=98 \%$, this trend is also recovered for this detail. The suction peak is too low for the L3, where the tip vortex is diffused too quickly due to the low mesh 


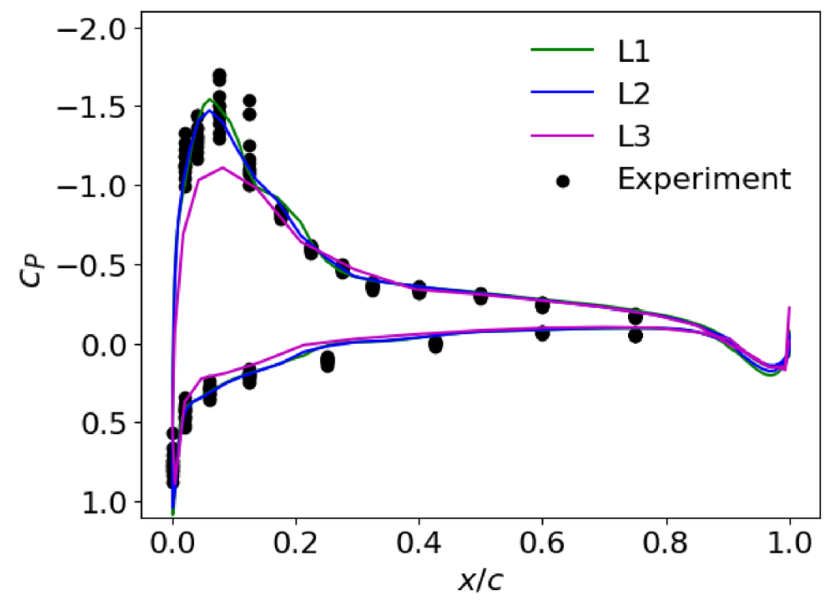

Fig. 4 Pressure distribution of $7 \mathrm{~A}$ rotor at $\mathrm{r} / \mathrm{R}=98 \%$

resolution. With increasing grid resolution, the suction peak of the simulations improves with respect to the experiment. See Fig. 5 for reference, where it is observed that the vortex passage is roughly at $r / R \approx 96 \%$. On the $\mathrm{L} 3$ mesh, the first and second tip vortex passages are barely distinct and the overall vorticity levels are low, while on the L1 mesh, a sharp first vortex passage at high vorticity is resolved.

\subsection{Forward flight/cruise}

This flight condition requires all four rotor blades to be modelled and, therefore, the application of Chimera. To

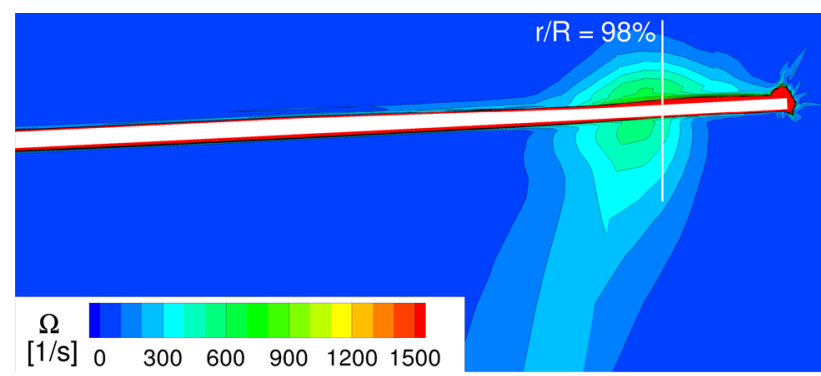

(a) L3 - coarse mesh

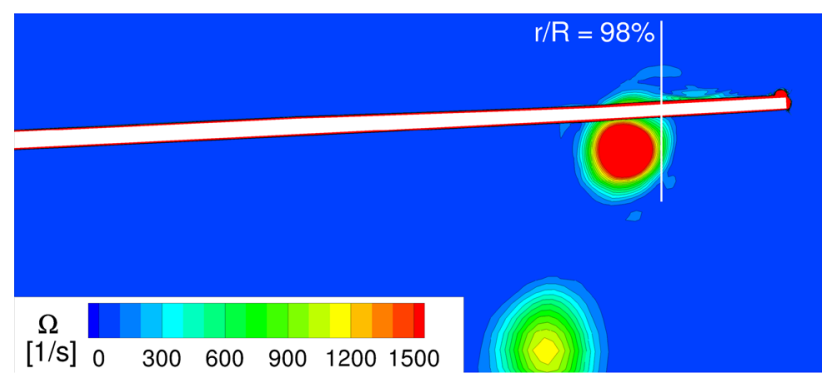

(b) L1 - fine mesh

Fig. 5 Vorticity plot cut through the rotor, looking from behind further improve the rotor trim, a crude fuselage is included to account for its displacement effect, see Fig. 2b.

Again, three levels are investigated. The L1 mesh consists of 129 grid points in chordwise, normal and radial direction for the blades, $161 \times 321 \times 401$ grid points in the vertical, spanwise and flight direction for the background grid, and 161x129x129 grid points for the fuselage mesh in longitudinal, normal and circumferential direction. This grants a total of 31 million grid points. The chosen time step is equivalent to $\Delta \psi=1 / 3^{\circ}$. For the L3 mesh, the fuselage is neglected as not enough overlap for the Chimera approach exists anymore. For the Chimera interpolation, an eighthorder Lagrangian interpolation [27] is utilized on the finest L1 grid, while for the coarser grids (L2 and L3) this is reduced to second-order linear interpolation as not enough overlap is given anymore.

For this flight condition, the data from the ERATO wind tunnel campaign [28] is chosen. Data point 373 belongs to the 7AD blade and data point 512 to the ERATO blade. Additionally, data point 142 for the 7AD blade from the Helishape is computed. The required power of the experiment along with the numerical results, is plotted in Fig. 6.

A similar trend to the hover simulation is observed, the L1 grid matches well with the experiment with a reasonable correlation on the $\mathrm{L} 2$ grid with data point 373 being an exception. Here the L2 grid underestimates the required power and the L1 results are even lower. The L3 grid strongly departs from the experiment for all data points. Considering all three data points, the relative changes among the rotors/flight conditions are still well reflected by the L1 and $\mathrm{L} 2$ grids.

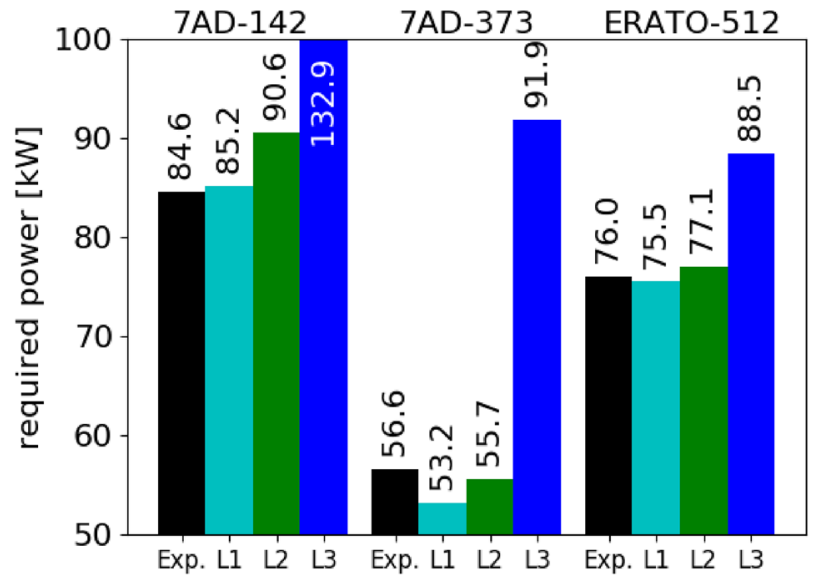

Fig. 6 Required power for the 7AD and ERATO rotor in forward flight 


\subsection{Descent}

For the descent flight condition, the same CFD setup is employed as for the cruise flight condition. This flight condition is rather challenging as it requires the correct capturing of the blade tip vortices. The parallel blade-vortex interaction (BVI) causes a quick variation of the angle-ofattack on the blades in the flight condition, which results in sharp pressure fluctuations giving the helicopter the distinct 'whop-whop' sound in descent flight.

For this scenario, results of the HART II baseline test case [29] are compared against the ERATO blade [28], data

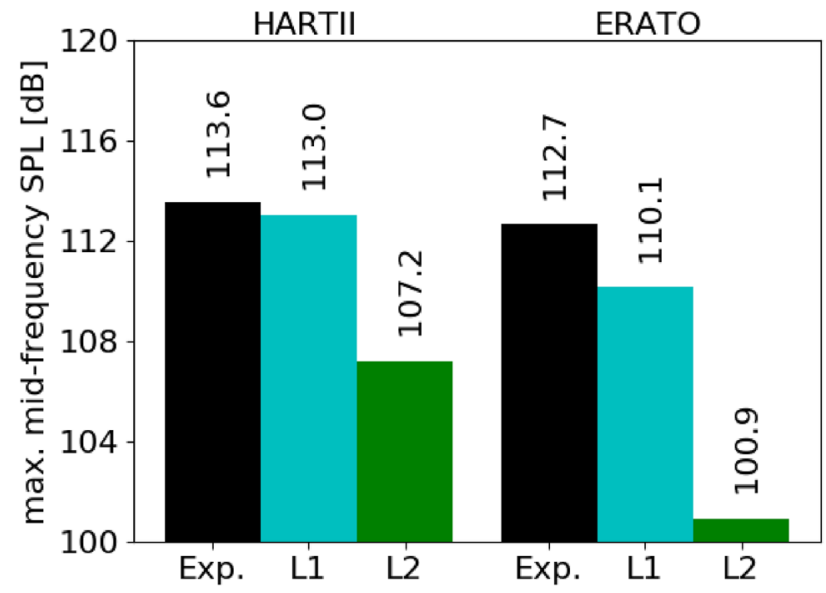

Fig. 7 Maximum mid-frequency (6-40 blade passing frequencies) SPL for different rotors point 399 . The maximum mid-frequency (6-40 blade passing frequencies) sound pressure level (SPL) found on the noise carpet is listed for each rotor in Fig. 7.

From this, it is found that all meshes deliver too quiet results relative to the experiments. This quietness is attributed to the still too diffusive nature of CFD on the relatively coarse grids. However, the tendency, that the HART II blade is louder than the ERATO blade is captured by all setups. To further understand the physics, the noise carpets for the HART II blade are shown in Fig. 8. From this, it is observed that the directivity is in good standing and the distribution of the left and right peak is correct with respect to the experiment. Further improvements of the directivity may be achieved including the fuselage in the acoustic simulation to account for shielding and scattering effects and eventually improved structural modeling.

From the investigation of these three flight conditions, it is decided to go with the $\mathrm{L} 2$ grid of each case as it provides a good trade-off between accuracy and speed.

\section{Surrogate-based optimization process}

The framework to control the optimization is discussed in greater detail in [11] and only a brief overview is given here. The general outline follows the ideas of the efficient global optimization by Jones et al. [30]. First a design of experiments is run, which is an organized random distribution of points in the parameter space to gain knowledge of the goal function and constraints. Here a central voronoi tessellated

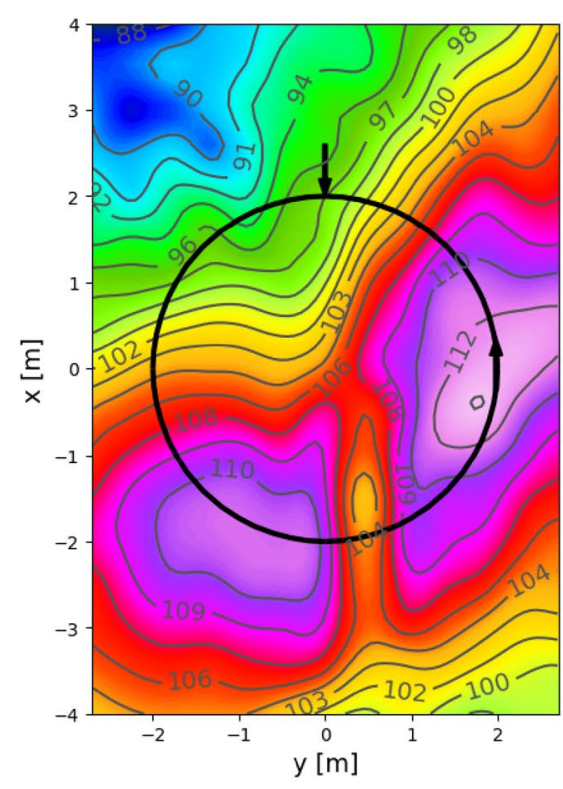

(a) Experiment

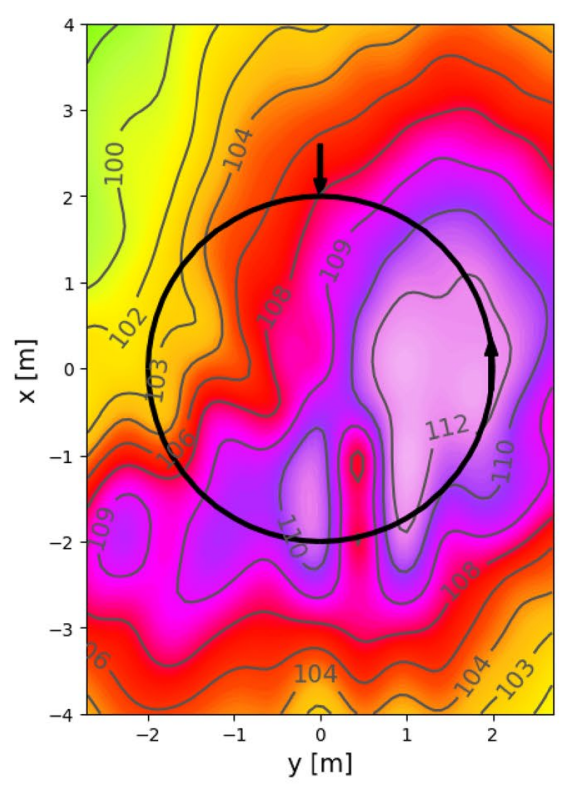

(b) Level 1

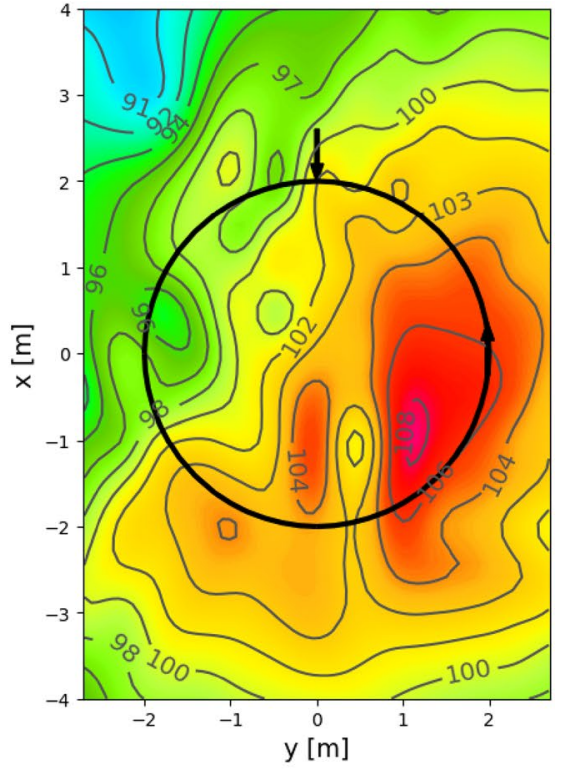

(c) Level 2

Fig. 8 HART II noise carpets for experiment and simulation in the mid-frequency range (6-40 blade passing frequencies) 
hypercube according to Romero et al. [31] is employed for good room filling properties. After calling the simulation, the surrogate models are built from the responses. A surrogate model is a simplification of the complex simulation function, and mimics the behavior of the simulation through much simpler numerical relations. Here, universal Kriging with regularization is used. In this scenario, a multi-objective optimization is carried out. Therefore, the differential evolutionary algorithm by Storn and Price [32] is applied here in combination with the sorting algorithm of the NSGA-II optimizer by Deb et al. [33]. It searches the Pareto front in the goal function surrogate models, while designs that violate the (surrogated) constraints are discarded. As this optimization process will find many designs, only a few are selected for evaluation with CFD. The designs that are furthest away from existing designs are selected to enrich the Pareto optimal set of simulated samples. An additional technique to avoid failing simulations is applied: if a design fails in a given flight condition as it may not be possible to trim the configuration, no value is added to the respective goal function and constraint surrogate models. However, an additional surrogate model is kept, which predicts whether a design will fail or not. All simulation locations are recorded here, with either a success or a fail. This way, regions with many failing designs are avoided, while no penalty function is necessary to taint the goal function models, keeping them pure.

The resources allocated for the optimization are visualized in Fig. 9. First, ten times the number of dimensions, here eight parameters, is utilized as the design of experiments (DoE). Then, for the three goals functions, three refinement cycles are carried out. Refinement cycles are defined as an optimization of each goal function individually, which is carried out in parallel under the consideration of all given constraints. The goal here is to identify the anchor points. A subset of three individuals is evaluated for each goal function at each refinement cycle. These consist of the best goal function, the highest expected improvement and the highest statistical error of the Kriging model found in the respective surrogate model. Note, for the goal function and highest statistical error, designs that violate the constraints are discarded and an alternative that fulfills all the constraint is sought instead. The expected improvement is multiplied by the probability that this point does not violate

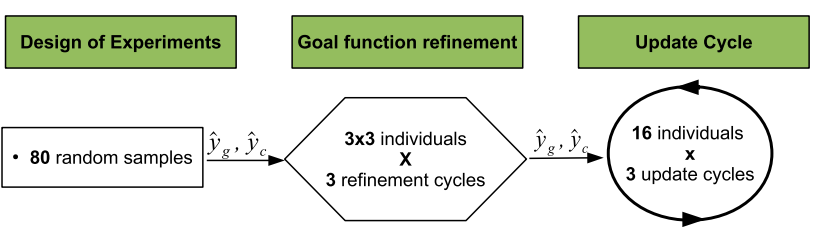

Fig. 9 Sketch of the surrogate based-optimization any constraints. Following the refinement cycle, the update cycle is initiated. During the update cycle, 16 individuals are extracted from the Pareto front search among the surrogate models. Again, constraint violating designs according to the surrogate models are ignored and only plausible designs are evaluated. Since the Pareto front of smooth functions could feature an infinite number of points, the points found from the multi-objective optimization are trimmed down in such a way that the distance between the resulting set is maximized and the doubling of existing points are avoided.

\section{Optimization task}

The chosen reference rotor is the renowned HART II blade, as it is well documented and data are openly available upon request [29]. The HART II blade has a rectangular planform with 2-meter radius, $0.121 \mathrm{~m}$ chord length and a constant twist gradient of $-8^{\circ} / R$. The trim settings along with respective goal functions and constraints that are evaluated with each flight condition are listed in Table 1. In hover and forward flight, the required power is minimized, while the maximum sound pressure level found on the experimental carpet is minimized in descent. Note, the controls $\theta_{0}, \theta_{c}, \theta_{s}$ and $\alpha$ refer to the collective, lateral and longitudinal cyclic pitch as well as the shaft angle. $c_{x} / \sigma, c_{z} / \sigma, c_{m x}, c_{m z}$ are the dimensionless coefficients for the propulsive and vertical force, as well as the pitch and roll moment. The constraint of eigenfrequency spacing is determined by the absolute minimal distance of any eigenmode of the blade to any multiple of rotational frequency. Up to seven eigenmodes are computed. The values of the constraints are chosen to be the ones obtained for the HART II rotor when computing them with the L2 setup. The environmental conditions have been chosen to match the HART II wind tunnel test

Table 1 Trim conditions of the individual flight conditions

\begin{tabular}{llll}
\hline & Hover & Cruise & Descent \\
\hline$M_{\text {tip }}$ & 0.641 & 0.641 & 0.641 \\
$M_{\infty}$ & 0. & 0.197 & 0.966 \\
$\gamma$ & $0^{o}$ & $0^{o}$ & $6^{o}$ \\
Controls & $\theta_{0}$ & $\theta_{0}, \theta_{c}, \theta_{s}, \alpha$ & $\theta_{0}, \theta_{c}, \theta_{s}$ \\
$c_{x} / \sigma$ & - & 0.001 & - \\
$c_{z} / \sigma$ & 0.1 & 0.095 & 0.056 \\
$c_{m x}$ & - & 0. & 0. \\
$c_{m y}$ & - & 0. & 0. \\
Goals & Req. power & & Max. SPL \\
(Minimization) & $114.6 \mathrm{~kW}$ & $94.2 \mathrm{~kW}$ & $105 \mathrm{~dB} \mathrm{~A}$ \\
Constraints & Eigenfreq. & Overflight & Peak root \\
& Spacing & Noise EPNL & Torsion \\
& $7.4 \%$ & $67.5 \mathrm{~dB}$ & $13.8 \mathrm{Nm}$ \\
\hline
\end{tabular}


for replicability, namely a temperature of $290.46^{\circ} \mathrm{K}$ and a pressure of 100, $970 \mathrm{~Pa}$. The descent flight condition is the baseline test case of the HART II wind tunnel campaign. While for a realistic rotor design, hot-and-high as well as one-engine-out (OEI) flight conditions are worth considering, this study focuses on the optimization of a wind tunnel rotor.

The here chosen parameterization focuses on the planform and twist of the rotor blade. In Fig. 10, a sketch of the quarter chord line properties of an example blade is plotted. Each planform curve is parameterized using cubic splines. The dots represent the control points of these splines, while the arrows show the free parameters for the optimizer and the direction in which they act. The ranges for each parameter are listed in Table 2. They are chosen in such a way that too many failing designs are avoided, even though the optimizer might choose the limit of certain parameters. The chord length is scaled in such a way as that the same thrust weighted rotor solidity is maintained as the HART II blade. It is noted that changing the planform parameters is also respected by the structural model in a simple way. The quarter chord line offset between the reference blade and the new design is applied to the center of gravity, neutral and elastic axis. The stiffness, mass, and inertia of the blade is scaled linearly with the chord length. It is acknowledged that more advanced approaches exists [12], yet have not been available for this work. In particular, the results of the descent flight condition might be subject to a larger changes as this flight condition is also sensitive to the blade motion.

\section{Results}

During the whole optimization, 151 rotor designs have been evaluated. Out of these 151 rotors, 1 rotor could not be trimmed in hover, 24 rotors failed in forward flight and 2 rotors failed in descent flight. Reasons for these failures

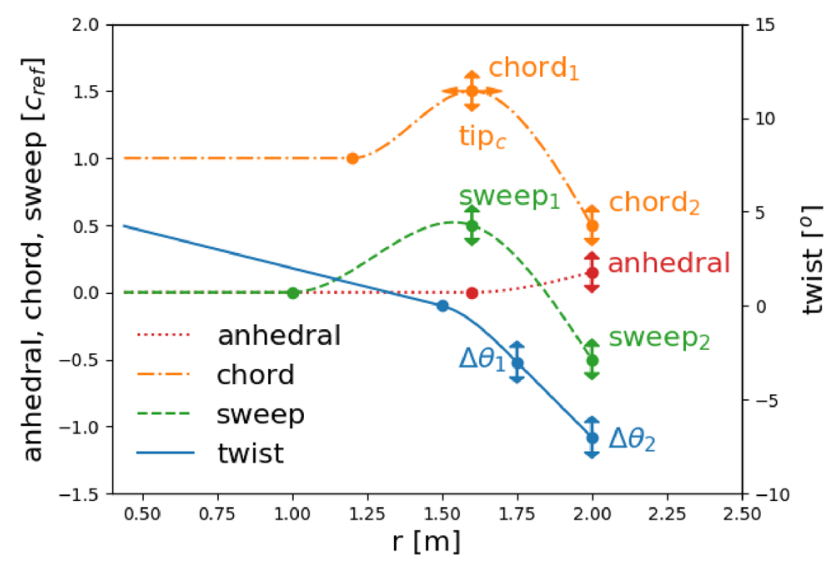

Fig. 10 Parameters of the optimization
Table 2 Parameter bounds of the optimization. $c_{r e f}=0.121 \mathrm{~m}$

\begin{tabular}{llll}
\hline Parameter & Lower bound & Upper bound & Unit \\
\hline Anhedral & -0.5 & 0.5 & $c_{\text {ref }}$ \\
tip $_{c}$ & 0.65 & 0.85 & $c_{\text {ref }}$ \\
chord $_{1}$ & 1. & 1.5 & $c_{\text {ref }}$ \\
chord $_{2}$ & 0.5 & 1. & $c_{\text {ref }}$ \\
sweep $_{1}$ & -0.5 & 0. & $c_{\text {ref }}$ \\
sweep $_{2}$ & 0. & 1.0 & $c_{\text {ref }}$ \\
$\Delta \theta_{1}$ & -5 & 5 & $o$ \\
$\Delta \theta_{2}$ & -10 & 0 & $o$ \\
\hline
\end{tabular}

range from not meeting the required thrust, the enforced pitch and rolling moments or the propulsive force, often caused by aero-elastic divergence. The resulting Pareto optimal set among these designs is shown in Fig. 11. The Pareto optimal set consists of 19 rotors, of which 12 improve in all goal functions with respect to the HART II baseline blade.

From these 19 rotors, five are selected to be analyzed further. The first three rotors represent the anchor points, which are the best in each goal function. Another two rotors are selected, which improved in all goal functions, but trade off between them. The respective merits of each of these selected designs are listed in Table 3, while a visualization is given in Fig. 12 and their respective parameter distribution is depicted in Fig. 13.

The best hover blade features a high twist gradient at the blade tip, with a moderate forward-backward sweep, a slight increase in chord length at first before it is tapered towards the tip. The most distinct feature is the small winglet/dihedral at the blade tip, which also reached its upper bound allowed in the optimization. Looking at Fig. 14, two things are noticed: first, the tip vortex is slightly weakened for this blade with respect to the baseline blade, while second, the

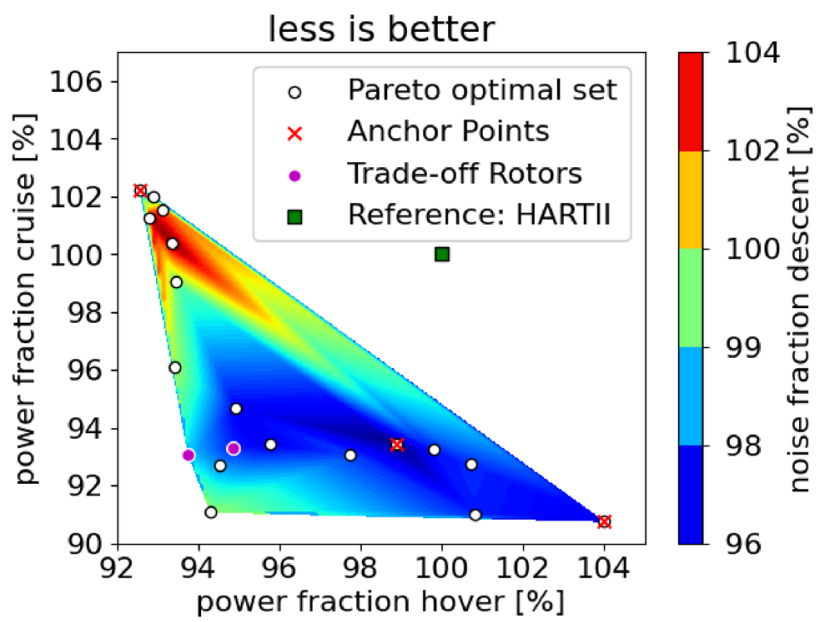

Fig. 11 Pareto front of the optimization 
Table 3 Goal function values of selected blades obtained from L2 setup

\begin{tabular}{llll}
\hline $\begin{array}{l}\text { Metric/ } \\
\text { Rotor }\end{array}$ & $\begin{array}{l}\text { Hover } \\
\text { Power (\%) }\end{array}$ & $\begin{array}{l}\text { Cruise } \\
\text { Power (\%) }\end{array}$ & $\begin{array}{l}\text { Descent } \\
\text { Noise } \\
(\%)\end{array}$ \\
\hline HART II & 100 & 100 & 100 \\
Best hover & 92.6 & 102 & 97.6 \\
Best cruise & 104 & 90.2 & 96.7 \\
Best descent & 98.9 & 92.8 & 96.1 \\
Trade-off hover & 93.8 & 92.7 & 98.1 \\
Trade-off cruise & 94.9 & 92.5 & 97.1 \\
\hline
\end{tabular}

trajectory passed over, instead of under the blade. This leads to an already observed vortex exploit effect [34]. Looking at Fig. 15, the effect on the thrust and torque distribution is seen. The thrust becomes more tri-angular in shape, a desirable attribute which may already be demonstrated with the blade element momentum theory. More surprisingly is the torque distribution of the optimized blade. The very outer part actually propels the blade slightly. The resulting force is tilted forward due to the large tip twist and aligned more into the vortex due to the dihedral. While this allows for an efficient hovering flight, the cruise flight power required grew with respect to the baseline blade, while it is slightly quieter in descent.

The best forward flight blade reduces the cruise power requirement drastically while sacrificing hover performance. The stronger forward-backward sweep also benefits the acoustic footprint, while the dihedral has been further reduced in contrast to the hover blade. Investigating the reason for the improvements, the new blade distributes the load more evenly over the disc and thus leads to reduced torque requirements overall, refer to Fig. 16. In particular, the tapered tip leads to less torque at the outer sections and more thrust produced at inner radial stations. In combination with the sweep, the transonic dip of the lift and thus torque is also reduced. It has to be mentioned though that the peak torsion has increased from $5.0^{\circ}$ for the HART II to blade to $6.3^{\circ}$, both occurring roughly at $\psi \approx 135^{\circ}$ azimuth.

The quietest descent blade has the advantage of improving in all goal functions. The winglet has mostly vanished for an even stronger blade sweep. This is not surprising as earlier studies revealed that neither an- nor dihedral are beneficial for the acoustic footprint [35], at least for this descent angle. The twist at the tip is actually reduced with respect to the baseline blade, while the chord length distribution is very similar to the baseline blade with a slight taper towards the tip. With the adverse twist gradient at the tip, the lift generation is shifted towards the rotational center. While this leads to some blade-vortex interaction more inboards, the outboard stations smear out the blade-vortex interactions

\section{Best hover}

top view

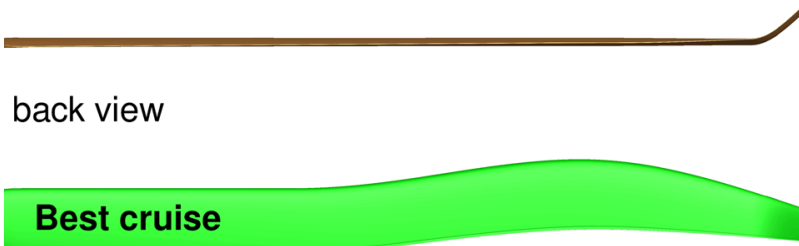

top view

\section{back view}

\section{Best descent flight}

top view

\section{back view}

\section{Trade-off hover}

top view

\section{back view}

\section{Trade-off cruise}

top view

\section{back view}

Fig. 12 Selected blades from the optimization

through the double sweep. Here the parallel interaction happens at different azimuth locations for different radial stations. The most forward leading edge will interact first, while the most backward leading-edge location last, thus not one strong event occurs per vortex, but smaller multiple or even continuous events. For reference, compare the sectional thrust derivative in Fig. 17. The smear out effect is primarily found on the advancing side. 


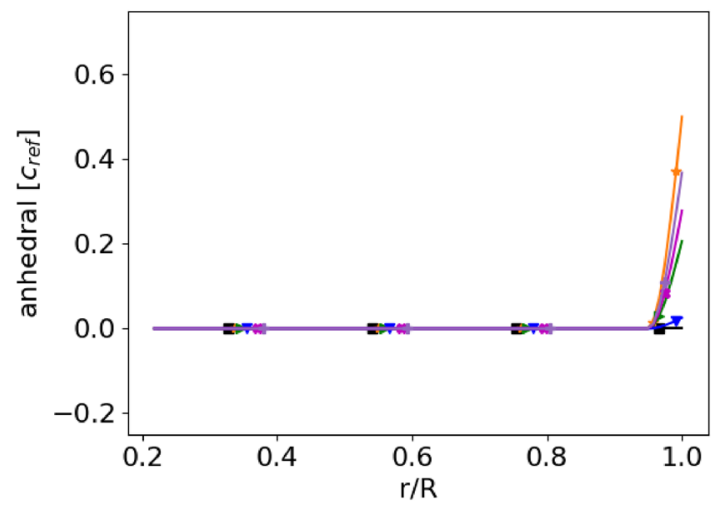

(a) anhedral

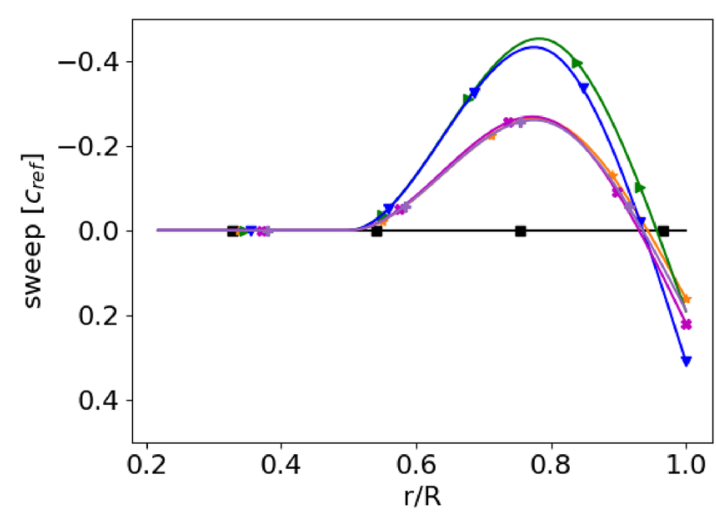

(c) sweep

Fig. 13 Parameter distribution of optimized blades

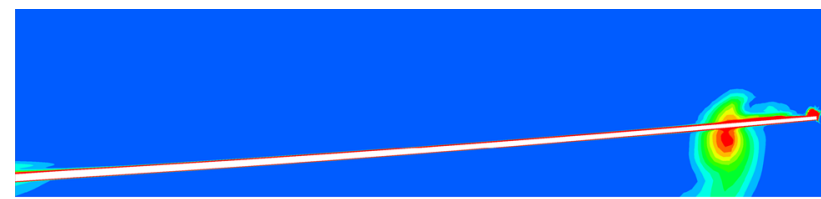

(a) HART II

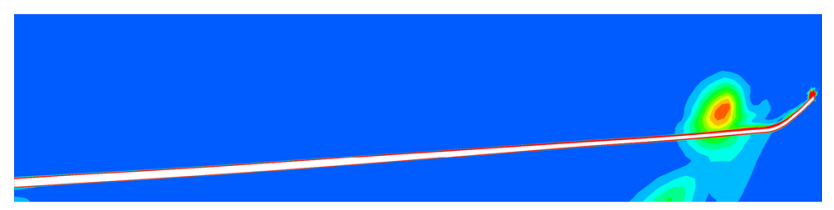

(b) Best hover

Fig. 14 Vorticity plot behind rotors in hover

The first trade-off blade improves quite well in all goal functions. It has a good hover and forward flight performance while also not becoming too loud in descent flight. The blade shape is in between the hover and forward flight blade. It exploits the previous tip vortex less in hover as the best hover blade does Fig. 15, yet features a similar tri-angular load. In forward flight, the thrust is also produced more

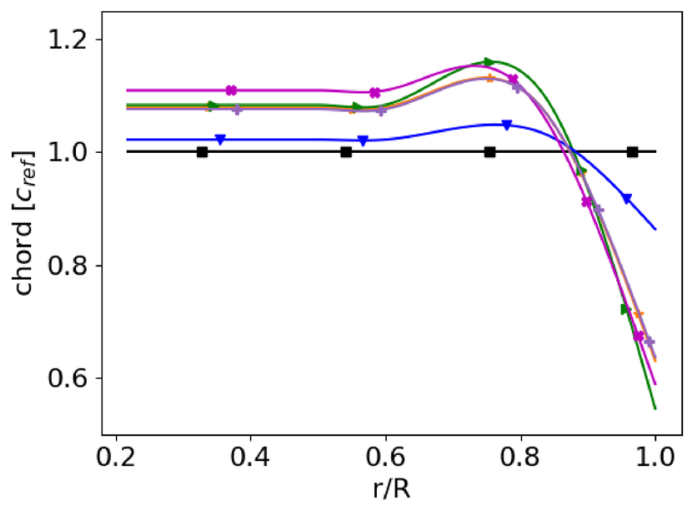

(b) chord

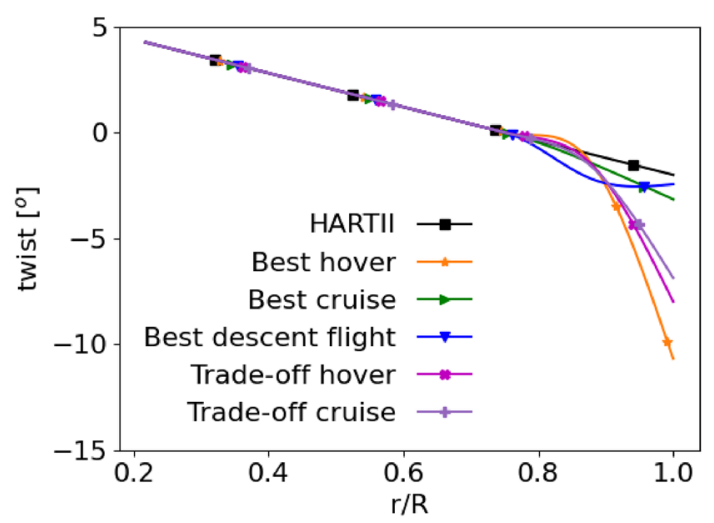

(d) twist

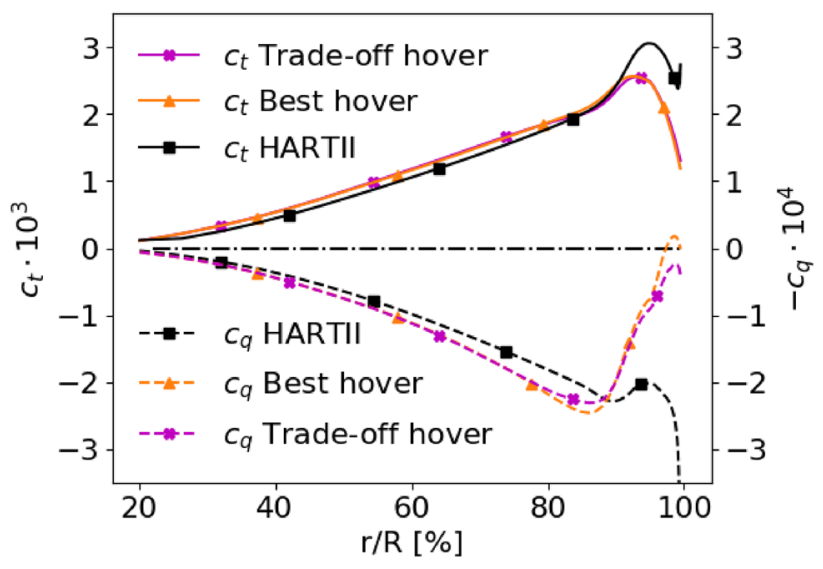

Fig. 15 Sectional thrust and torque coefficient of rotors in hover

inside, however, due to the smaller chord length $r / R \approx 75 \%$, the airfoils align less optimal and more torque is produced in this area than for the best forward flight blade, Fig. 16. In descent, the smearing out of the advancing side blade vortex interaction events is also observed. However, the overall lift 


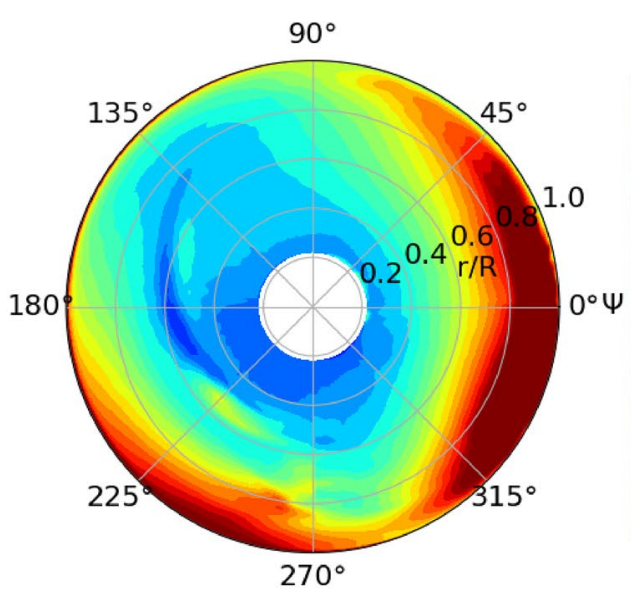

(a) HART II

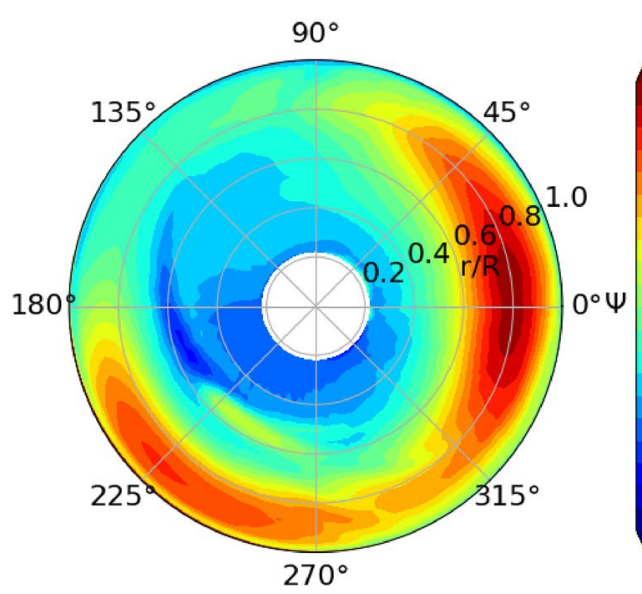

$c_{q} \cdot 10^{4}$

(b) Best cruise

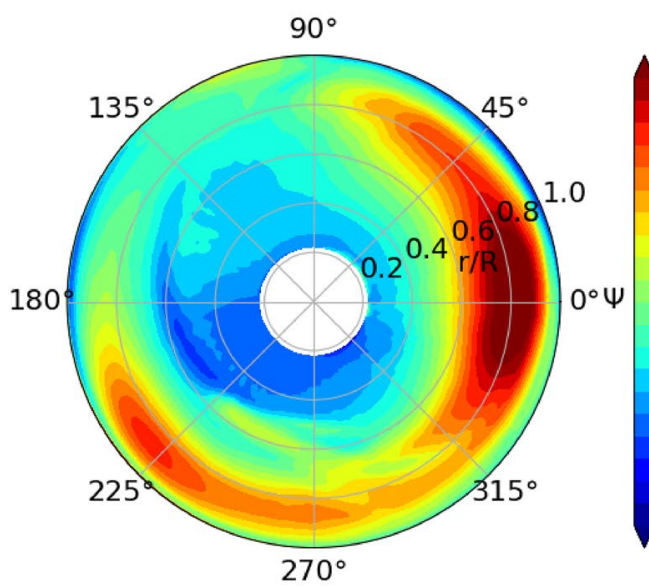

$c_{q} \cdot 10^{4}$

(c) Trade-off hover

Fig. 16 Sectional torque in forward flight

distribution is a bit more outboard in comparison with the best descent flight blade due to the regular negative twist at the tip. Overall, this trade-off blade also shows that the

$c_{q} \cdot 10^{4}$

2.4

1.8

1.2

0.6

0.0

$-0.6$

2.4

1.8

1.2

0.6

0.0

$-0.6$

3.0

2.4

1.8

1.2

0.6

0.0

$-0.6$

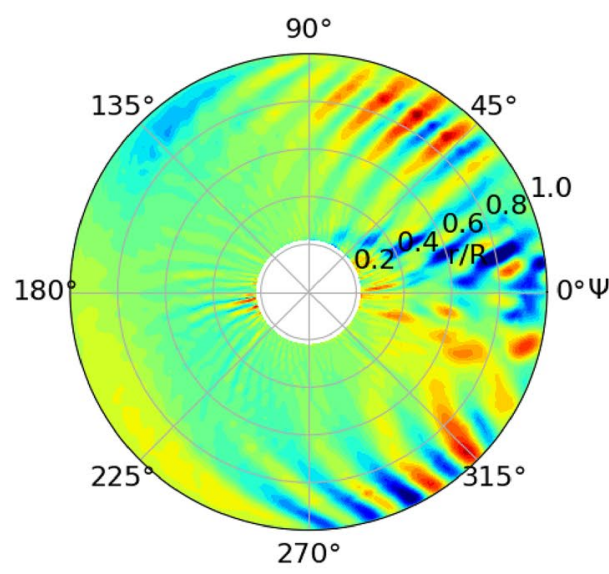

$d c_{t} N / d \psi$

(a) HART II

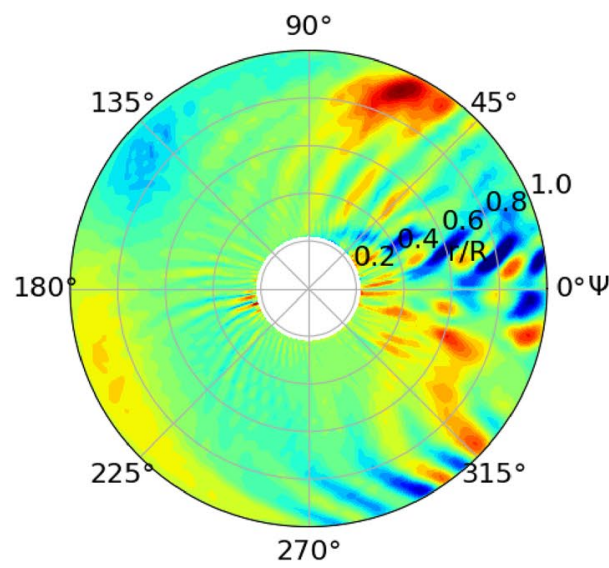

$d c_{t} N / d \psi$ $\cdot 10^{5}\left[1 /{ }^{\circ}\right]$

(b) Best descent

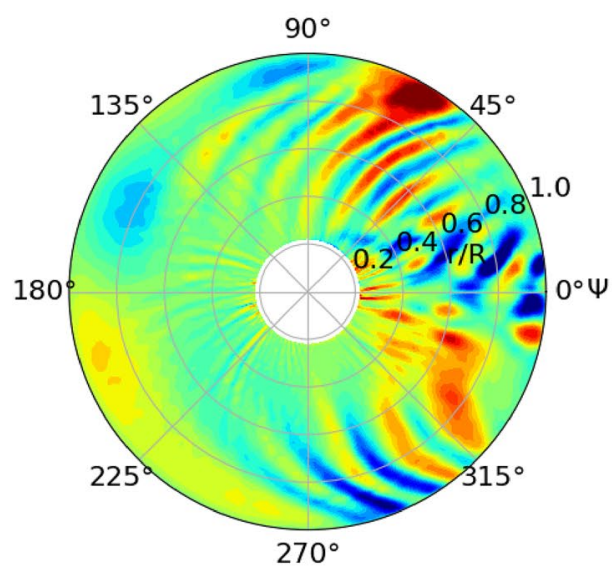

$d c_{t} N / d \psi$ $\cdot 10^{5}\left[1 / /^{\circ}\right]$

(c) Trade-off hover

Fig. 17 Sectional thrust derivative in descent flight 
beneficial effects seen for the Pareto front anchors are found again, yet are not as pronounced as the parameters are in between the anchor values.

The second trade-off blade is slightly quieter in descent and better in the cruise flight condition than the first tradeoff blade, while sacrificing some hover performance with respect to the first trade-off blade. This blade also underlines that this region of the Pareto front is most likely continuous, as small geometrical changes with respect to the first tradeoff blade allowed for small adjustments in the goal functions. Again, the physical phenomena driving this blade are very similar to the first trade-off blade, with a slight shift towards better forward flight and less hover performance in comparison with the hover biased trade-off blade.

\section{Off-design analysis}

After the discussion of the selected blades with respect to their improvements in the individual goal functions, an offdesign analysis of the blades is performed to further investigate the robustness of each blade design. The hover polar, figure of merit over thrust, is plotted in Fig. 18, while a shaft angle sweep in descent flight is plotted in Fig. 19 for the peak A-weighted noise. In Fig. 20 and Fig. 21, the absolute and relative forward flight power requirements are plotted over speed. On the top, the absolute values are shown, while on the bottom the relative change with respect to the reference HART II blade are plotted

The hover polar shows the results up to the data points past one data point of the maximum figure of merit with a spacing of $\Delta c_{T} / \sigma=2 \%$. From this plot, it is seen that for the forward flight blade the point of the maximum figure of merit is the previous one to the design point. From this, it is concluded that no reserves for this rotor exists and therefore

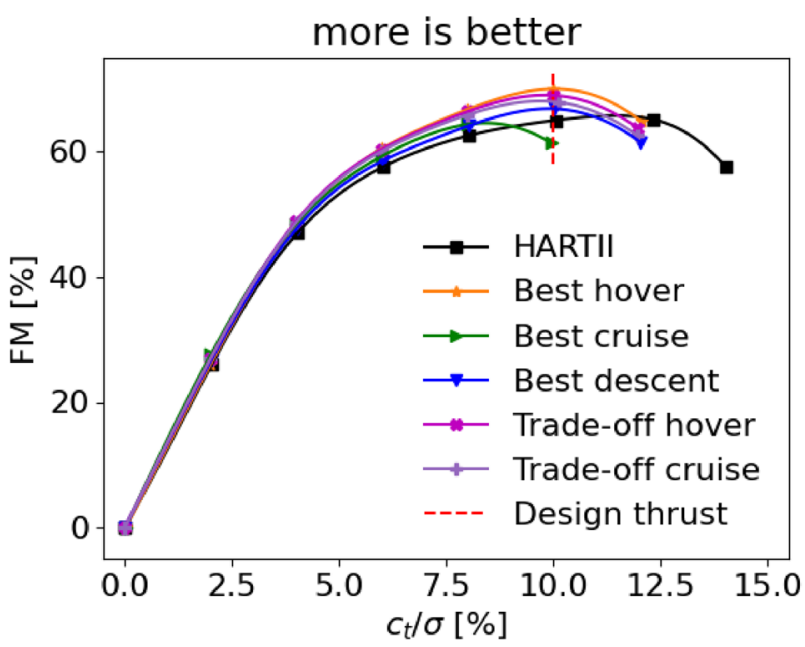

Fig. 18 Hover polar

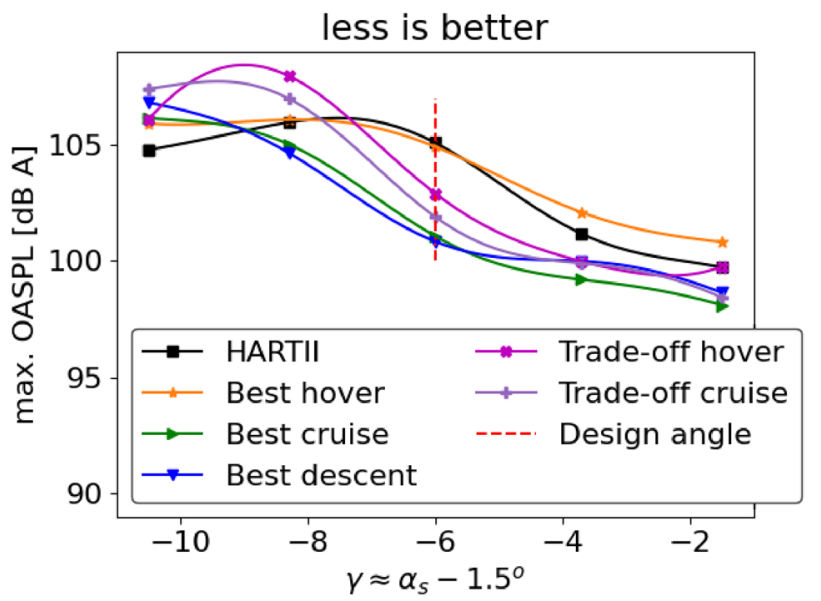

Fig. 19 Descent polar

it is considered an infeasible design. For the other blades, all of them improve over the baseline blade at the design point, which is also the maximum Figure of Merit for these points. This behavior is different from what has been observed in the GRC project, where the overall polar improved for the (hover) optimized blades. This is likely attributed to the constant solidity, which has been enforced here, but not in the GRC project. In the GRC project, the better hover blades increased the solidity slightly giving them more head space at higher thrust settings. In this optimization, the thrust weighted solidity remained constant and thus adding more sweep or twist will potentially limit the maximum thrust, but also lead to a design tailored more towards the design point. The upside is that all blades improved over the baseline blade for lower thrust settings.

The descent polar is generated by simply changing the shaft angle and re-trimming the rotor to the new flow condition. This implies that the same wind tunnel correction of $1.5^{\circ}$ is applied to all shaft angles, which may lose its validity further away from the design point. From this plot, it is seen that all blades improve in the design descent condition and more shallow descent flights; however, at the steepest descent angle, the baseline blade becomes the quietest blade. For the second steepest descent angle, only the descent and cruise blades are quieter than the baseline blade, with the hover blade being at a similar level and the trade-off blades being louder. Looking at the vortex field of the HART II and cruise biased trade-off blade in the steepest flight condition, Fig. 22, it is observed that the tip vortices are further away and weaker for the HARTII blade than they are for the tradeoff blade. This leads to a stronger blade-vortex interaction for the cruise biased trade-off blade than for the HART II blade, also leading to more noise produced. This effect is partially amplified from the dihedral, which gives the releasing vortex a spin towards the inboard sections, which is more severe for the steeper descent angles than for the shallow 


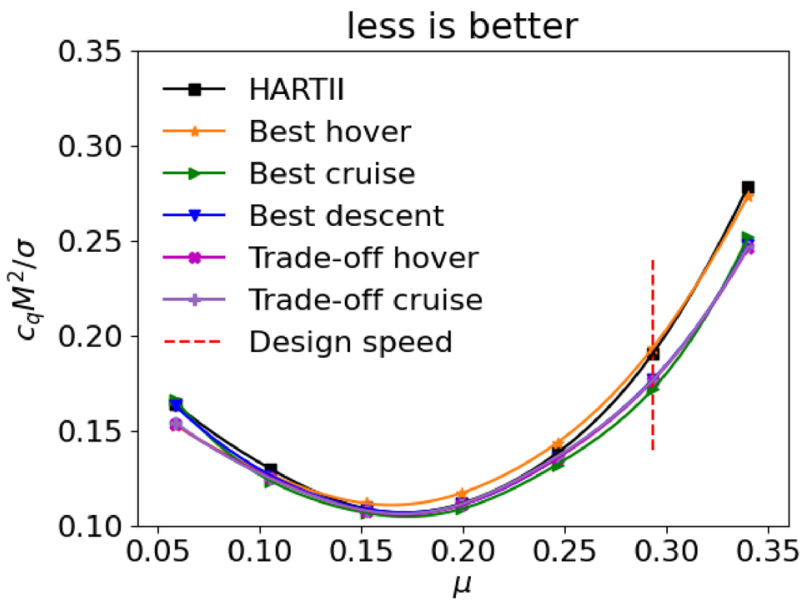

Fig. 20 Forward flight polar

ones. This problem might be avoided by optimizing blades at multiple descent angles and averaging the results together to overcome this in the future. This will yet have to be shown. Also, the structural adjustment plays an important role in this flight condition as a changed mass distribution of the

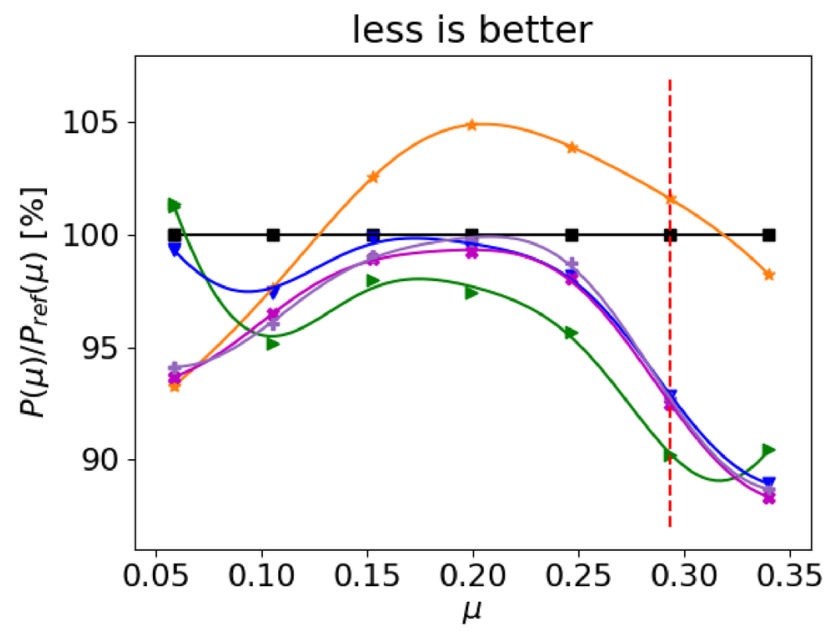

Fig. 21 Relative forward flight polar w.r.t. HART II blade

blade will alter the flapping behavior and may improve the vortex miss distance.

In forward flight, all blades except the hover blade improve for advance ratios greater $10 \%$. Waviness in the relative plot is observed, which may be due to the trim convergences criterion of $0.5 \%$ relative change in power from one coupling step to the next. Thus, the curve fit is slightly regressed. The overall trend seems to be in good standing though, when looking at the absolute values. All optimized blades surpass the reference blade at the fastest speed, which could potentially mean that the envelope could be opened

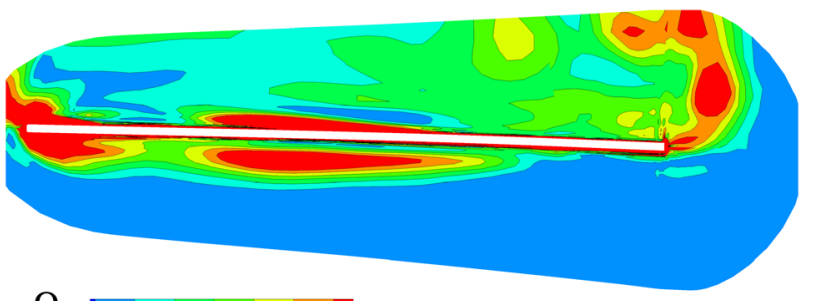

$\Omega$

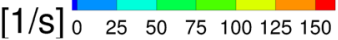

(a) HART II

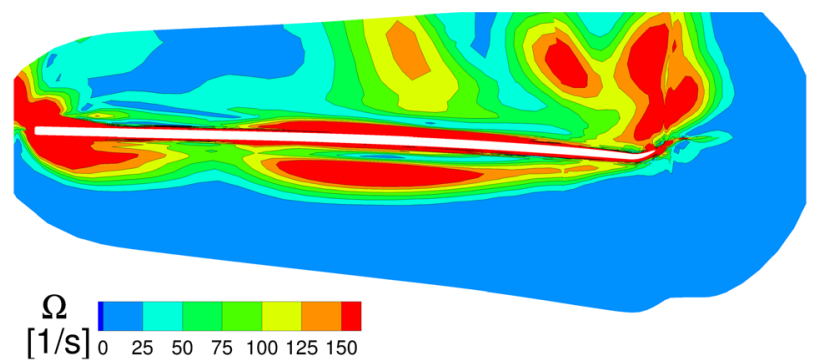

(b) Trade-off cruise

Fig. 22 Vorticity plot behind advancing side blade $\left(\psi=70^{\circ}\right.$, only blade mesh solution shown)

to higher speeds if vibratory loads remain in acceptable limits. The hover blade requires more power in the intermediate advance ratios which also means that the ability to climb is reduced. All other blades should maintain similar climb and one-engine out performance except for the cruise blade, for which this improves. The descent and cruise blade are close and worse than the reference blade at the lowest advance ratio. This is not surprising as the forward flight blade requires more power in hover and the descent flight blade only shows a minor improvement.

\section{Feasibility of the approach}

A brief section is dedicated to the feasibility of the retrieved designs and overall optimization approach. While already incorporating three different flight conditions, three goal functions and three constraints, the final rotor blades still have to be checked in many more flight conditions for its feasibility. On the one hand, vibratory loads have not been addressed in this context, while on the other hand the rather crude linear structural scaling is likely not viable to obtain the final blade structural design. Therefore, the here shown blades should be considered a proposal of the aerodynamics and aero-acoustics department, but need to be thoroughly checked, improved and potentially further constraint by the structural dynamics team. They may even be discarded or have the here utilized aerodynamic driven parameters changed to fulfill all necessary requirements of an actual, manufacturable helicopter rotor blade. Here the obtained 
surrogate models could aid in identifying further trade-offs as they allow to quickly estimate the new merits for the altered blade.

Also, considering the results of the off-design analysis, it is likely required already on the aerodynamic and aeroacoustic side to include more flight conditions to ensure more robust designs. For example, one engine out flight conditions, hot-and-high performance could be additionally assessed, while for descent flight an uncertainty approach, such as has been shown by Sabater et al [36] for fixedwing aircraft, could be applied to ensure that designs are less sensitive to the precise numbers of the flight condition. Unfortunately, the computational cost necessary to perform such a task, even with the availability of surrogate models and high-performance computing clusters, quickly becomes prohibitively expensive. Eventually the numbers of possible parameters to be optimized need to be traded for the numbers of flight conditions investigated.

\section{Summary}

The following achievements have been presented in this paper:

- The novel simulation setup using higher order CFD and laminar-turbulent transition prediction has been validated against experimental data in hover, forward and descent flight for different rotors. The agreement with the experiment for the required power in hover and forward flight was good, while in descent flight the noise trend was well matched. For descent flight, it was shown that with increasing number of resources the absolute values can also be matched better, however these resources prove to be too expensive to be used during the optimization

- The multi-objective optimization has been carried out with three different goal functions and three different constraints coming from three flight conditions using eight design variables. A total of 151 rotors have been evaluated of which 19 showed to be Pareto optimal and 12 improved in each goal function with respect to the baseline blade. The best blades in each goal function have been examined in more detail along with two tradeoff blades of the Pareto front.

- An off-design analysis has been performed for the selected blades. From this analysis it has been found that in descent flight, an overall improvement cannot be achieved using individual design points for the optimization.

On the one hand, this shows that the Pareto front approach is viable for the design of rotor blades, while on the other hand more computational resources are necessary to include more flight conditions. Even though the higher order methods allowed for efficient simulation setups, the fidelity of the results could be further increased by employing more grid points. This, however, will likely require the next HPC generation being available in 5-10 years.

To design next generation rotor blades, as is planned in DLR's UrbanRescue project, the following steps should be taken:

- Optimization of airfoils for helicopters: Since the aerodynamic and aeroacoustic optimization of helicopter rotor blade planform and twist is on a very mature level methodically, the inclusion of airfoil optimization is the next step to enhance the aerodynamic performance

- Structural dynamic considerations: While the current approach included a first metric to check the blade dynamics, a more advanced approach is necessary. The blade structural properties should also be optimized to tune vibratory loads and avoid excitation of natural eigenfrequencies of the rotor. Examples of this can be found in the literature by Glaz et al. [37]

- Both, the aerodynamic and structural dynamic optimization requires a reliable feedback on the feasibility of structural properties. It is unsure, whether the blade internal structure can be produced, nor if it holds the outer aerodynamic loads. An approach similar to the one by Airbus Helicopters France [12] could be used.

Funding Open Access funding enabled and organized by Projekt DEAL.

Open Access This article is licensed under a Creative Commons Attribution 4.0 International License, which permits use, sharing, adaptation, distribution and reproduction in any medium or format, as long as you give appropriate credit to the original author(s) and the source, provide a link to the Creative Commons licence, and indicate if changes were made. The images or other third party material in this article are included in the article's Creative Commons licence, unless indicated otherwise in a credit line to the material. If material is not included in the article's Creative Commons licence and your intended use is not permitted by statutory regulation or exceeds the permitted use, you will need to obtain permission directly from the copyright holder. To view a copy of this licence, visit http://creativecommons.org/licenses/by/4.0/.

\section{References}

1. Undertaking, Clean Sky Joint. The Clean Sky JTI (Joint Technology Initiative), (2012)

2. Görtz, S., Krumbein, A., Ritter, M., Hofmann, J.: DLR-Projekt VicToria - Virtual Aircraft Technology Integration Platform. In Deutscher Luft- und Raumfahrtkongress, (2018) 
3. Kowarsch, U., Öhrle, C., Keßler, M., Krämer, E.: Aeroacoustic Simulation of a Complete H145 Helicopter in Descent Flight. J. Am. Helicop. Soc. 61(4), 0420011-04200113 (2016)

4. Ganguli, R.: Survey of Recent Developments in Rotorcraft Design Optimization. J. Aircraft 41(3), 493-510 (2004)

5. Fabiano, E., Mavriplis, D.: Adjoint-Based Aeroacoustic DesignOptimization of Flexible Rotors in Forward Flight. J. Am. Helicop. Soc. 62-4(042005), 1-17 (2017)

6. Wang, L., Diskin, B., Biedron, R.T., Nielsen, E.J., Eric, Bauchau O.A: High-Fidelity Multidisciplinary Sensitivity Analysis and Design Optimization for Rotorcraft Applications. AIAA J. 57(8), 3117-3131 (2019)

7. Jimenez-Garcia, A., Biava, M., Barakos, G.N., Baverstock, K.D., Gates, S., Mullen, P.: Tiltrotor CFD Part II - aerodynamic optimisation of tiltrotor blades. Aeronaut. J. 121(1239), 611-636 (2017)

8. Roca León, E., Le Pape, A., Costes, M., Desiderie, J-A., Alfano, D.: Concurrent Aerodynamic Optimization of Rotor Blades Using a Nash Game Method. J. Am. Helicop. Soc., (2016)

9. Johnson, C., Barakos, G. N.: A Framework for the Optimization of a BERP-like Blade. In 38th European Rotorcraft Forum

10. Leusink, D., Alfano, D., Cinnella, P.: Multi-fidelity optimization strategy for the industrial aerodynamic design of helicopter rotor blades. Aerospace Sci. Technol. 42, 136-147 (2015)

11. Wilke, G.: Variable-Fidelity Methodology for the Aerodynamic Optimization of Helicopter Rotors. AIAA J. 57(8), 3145-3158 (2019)

12. Desvigne, D., Coisnon, R., Michel, B. R., Thomas, A., Pinacho, J. P., Roca León, E.: Multi-Objective Industrial Optimization of High-Speed Helicopter Main Rotor Blades with DynamicallyAdapted Structural Properties. In 45th European Rotorcraft Forum, (2019)

13. Smith, M. J., Lim, J. W., van der Wall, B. G., Baeder, J. D., Biedron, R. T., Boyd Jr, D. D., Jayaraman, B., Jung, S. N., Min, B-Y: An assessment of CFD/CSD prediction state-of-the-art using the HART II international workshop data. In 68th Annual Forum of the American Helicopter Society, Ft. Worth, TX, (2012)

14. Allen, C.B.: Chimera volume grid generation within the eros code. Proc. Inst. Mechan. Eng. Part G J. Aerospace Eng. 214, 125-140 (2000)

15. Benoit, B., Dequin, A.-M., Kampa, K., von Grünhagen, W., Basset, P.-M., Gimonet, B.:HOST, a General Helicopter Simulation Tool for Germany and France. In 56th Annual Forum of the American Helicopters Society, Virginia Beach, VA, May (2000)

16. Potsdam, M., Yeo, H., Johnson, W.: Rotor Airloads Prediction Using Loose Aerodynamic/Structural Coupling. In American Helicopter Society 60th Annual Forum, (2004)

17. Raddatz, J., Fassbender, J. K.: Block Structured Navier-Stokes Solver FLOWer. In Norbert Kroll and Jens K. Fassbender, editors, MEGAFLOW - Numerical Flow Simulation for Aircraft Design, pages 27-44, Berlin, Heidelberg, (2005)

18. Kitamura, K., Shima, E.: Towards shock-stable and accurate hypersonic heating computations: A new pressure flux for AUSMfamily schemes. J. Comput. Phys. 245, 62-83 (2013)

19. Yamamoto, S., Kano, S., Daiguji, H.: An efficient CFD approach for simulating unsteady hypersonic shock-shock interference flows. Computers \& Fluids 27(5), 571-580 (1998)

20. Dacles-Mariani, J., Zilliac, G.G., Chow, J.S., Bradshaw, P.: Numerical/experimental study of a wingtip vortex in the near field. AIAA J. 33(9), 1561-1568 (1995)

21. Heister, C.C.: A Method for Approximate Prediction of LaminarTurbulent Transition on Helicopter Rotors. J. Am. Helicop. Soc. 63(3), 1-14 (2018)
22. Schwarz, T.: Ein blockstrukturiertes Verfahren zur Simulation der Umströmung komplexer Konfigurationen. $\mathrm{PhD}$ thesis, Institut für Aerodynamik und Strömungstechnik, DLR, Braunschweig, (2005)

23. Yin, J., Delfs, J.: Improvement of DLR Rotor Aeroacoustic Code (APSIM) and its Validation with Analytic Solution. In 29th European Rotorcraft Forum, (2003)

24. Di Francescantonio, P.: A new Boundary Integral Formulation for the Prediction of Sound Radiation. J. Sound Vibr. 202-4, 491-509 (1997)

25. Brentner, K.S., Farassat, F.: Modeling aerodynamically generated sound of helicopter rotors. Progress. Aerospace Sci. 39-2, 83-120 (2003)

26. Schultz, K. J., Splettstößer, W., Junker, B., Wagner, W., Schoell, E., Mercker, E., Pengel, K., Arnaud, G., Fertis, D.: A Parametric Wind Tunnel Test on Rotorcraft Aerodynamics and Aeroacoustics (HELISHAPE). Test Procedures and Representative Results. In 22nd European Rotorcraft Forum, (1996)

27. Wriggers, P.: Nichtlineare Finite-Element-Methoden. SpringerVerlag, Berlin Heidelberg (2001)

28. van der Wall, B.G., Kessler, C., Delrieux, Y., Beaumier, P., Gervais, M., Hirsch, J-F, Pengel, K., Crozier, P.: From ERATO Basic Research to the Blue Edge Rotor Blade. In AHS International 72nd Annual Forum \& Technology Display, (2016)

29. van der Wall, B.G.: A comprehensive rotary-wing data base for code validation: The hart ii international workshop. Aeron. J. 115(1164), 91-102 (2011)

30. Jones, D.R., Schonlau, M., Welch, W.J.: Efficient Global Optimization of Expensive Black-Box Functions. J. Global Optimiz. 13, 455-492 (1998)

31. Romero, V.J., Burkardt, J.V., Gunzburger, M.D., Peterson, J.S.: Comparison of pure and "Latinized" centroidal Voronoi tessellation against various other statistical sampling methods. Reliab. Eng. Syst. Safety 91, 1266-1280 (2006)

32. Storn, R., Price, K.: Differential Evolution - A simple and efficient adaptive scheme for global optimization over continuous spaces. J. Global Optimiz. 11, 341-359 (1997)

33. Deb, K., Pratap, A., Agarwal, S., Meyarivan, T.: A fast and elitist multiobjective genetic algorithm: NSGA-II. IEEE Trans. Evolution. Comput. 6(2), 182-197 (2002)

34. Wilke, G.: Aerodynamic Optimization of Helicopter Rotorblades using Variable Fidelity Methods. $\mathrm{PhD}$ thesis, Technische Universität Braunschweig, (2017)

35. Wilke, G.: Findings in aero-acoustic simulations for optimizations. In Proceedings of the 76th Annual Forum, Virtual, October (2020). The Vertical Flight Society

36. Sabater, C., Bekemeyer, P., Philipp, Görtz, S.: Efficient bilevel surrogate approach for optimization under uncertainty of shock control bumps. AIAA J., 0(0):1-15, (2020)

37. Glaz, B., Goel, T., Liu, L., Friedmann, P.P., Haftka, R.T.: Multiple-Surrogate Approach to Helicopter Rotor Blade Vibration Reduction. AIAA J. 47(1), 271-282 (2009)

Publisher's Note Springer Nature remains neutral with regard to jurisdictional claims in published maps and institutional affiliations. 